\title{
A New VISTA on combination therapy for negative checkpoint regulator blockade
}

\author{
Jie Deng ${ }^{1}$, Isabelle Le Mercier ${ }^{1,2^{*}}$, Anna Kuta ${ }^{1}$ and Randolph J. Noelle ${ }^{1,2^{*}}$
}

\begin{abstract}
Negative checkpoint regulators function to restrain $T$ cell responses to maintain tolerance and limit immunopathology. However, in the setting of malignancy, these pathways work in concert to promote immunemediate escape leading to the development of a clinically overt cancer. In the recent years, clinical trials demonstrating the efficacy of blocking antibodies against these molecules have invigorated the field of immunotherapy. In this review, we discuss the current understanding on established NCR blockade and how strategic combination therapy with anti-VISTA antibody can be used to target multiple non-redundant NCR pathways.
\end{abstract}

Keywords: Negative checkpoint regulators, Combination immunotherapy, VISTA

\section{Background}

Negative checkpoint regulator (NCR) blockade has begun to establish itself as a cornerstone to multi-modality cancer treatment. Pioneered by seminal findings in multiple mouse models and human cancers, monoclonal antibody blockade of Cytotoxic T Lymphocyte Antigen 4 (CTLA-4) has paved the way for the field of NCRs leading to discovery of new molecules as well as uncovering novel approaches for combination therapy targeting multiple non-redundant pathways. In this review, we describe the prevailing wisdom of mechanisms of action for established NCR blockade. Further, we discuss the role of V-domain Ig Suppressor of T Cell Activation (VISTA), a novel target in immuno-oncology. Alongside the phase 1 clinical trial testing anti-VISTA (NCT02671955), it becomes increasingly important to have a comprehensive understanding of how targeting VISTA advantageously shapes the tumor microenvironment.

Blockade of CTLA-4 paving the way for checkpoint blockade CTLA-4 is exclusively expressed on T cells, although not on naïve or memory T cells. CTLA-4 has two binding partners, B7 molecules CD80 and CD86, both of which

\footnotetext{
* Correspondence: lemercier@immunext.com;

Randolph.J.Noelle@dartmouth.edu

${ }^{1}$ Department of Microbiology and Immunology, Geisel School of Medicine at Dartmouth, Norris Cotton Cancer Center, One Medical Center Drive, Room

730, Lebanon, NH 03756, USA

Full list of author information is available at the end of the article
}

are expressed on antigen presenting cells (APCs). CTLA-4 exerts its immunosuppression of $\mathrm{T}$ cell responses by multiple mechanisms. First, CTLA- 4 outcompetes the co-stimulatory molecule $\mathrm{CD} 28$ for their shared binding partners CD80 and CD86 as a result of increased affinity, increased avidity, and more stable interactions within the immunological synapse [1-6]. In addition, upon disrupting co-simulation by $\mathrm{CD} 28$ and ligation with cognate $\mathrm{B} 7$ molecules, CTLA- 4 recruits the inhibitory tyrosine phosphatase SHP-2 to the T cell receptor, thus attenuating the signal $[1,2,7]$, destabilizing the immunological synapse $[1,8]$, arresting cell cycling [9], and reducing the production of $\mathrm{IL}-2[1,2,7,9]$. CTLA-4 can also signal bi-directionally to induce the upregulation of indoleamine 2,3-dioxygenase (IDO) by APCs $[10,11]$. In vivo, it is hypothesized that the predominant mechanism through which CTLA-4 downregulates $\mathrm{T}$ cell response is via transcytosis of CD80 and CD86 from the membranes of APCs, thus making these molecules unavailable for co-stimulation by CD28 $[1,8$, 12]. Furthermore, natural FoxP3 ${ }^{+}$regulatory $\mathrm{T}$ cells (nTreg) constitutively express high levels of CTLA-4 on their surface and both CTLA-4 on nTregs as well as conventional $\mathrm{T}$ cells contribute to $\mathrm{T}$ cell immunosuppression [13].

The major role CTLA-4 plays in restraining T cell responses to maintain tolerance is evident in mice that have a complete deficiency of CTLA-4 $[1,9,14,15]$. 
Within a few weeks, CTLA-4 $4^{-/-}$mice die from massive, spontaneous, lethal autoimmunity due to unchecked lymphoproliferation [14, 15]. Activated T cells aggressively infiltrated spleen and lymph nodes as well as the liver, heart, lung and pancreas with high immunoglobulin detected in the blood [14, 15]. This severe and widespread autoimmune phenotype suggests that CTLA-4 primarily functions to control T cell responses for maintaining immune homeostasis. Importantly, the absence of CTLA-4 specifically on Tregs is sufficient for the development of autoimmunity, further reiterating the major role of CTLA- 4 in Treg mediated suppression [16].

These observations that CTLA-4 functions to restrain $\mathrm{T}$ cell response led to the hypothesis that blockade of CTLA-4 may lead to persistent T cell activity within tumors. Success in multiple pre-clinical models [17, 18], led to the development of Ipilimumab (Bristol-Myers Squibb), a fully human IgG1 monoclonal antibody and first in class of NCR blockade. After two transformative phase III trials in melanoma [19, 20], Ipilimumab received FDA approval in 2011 for the treatment of advanced melanoma. Further, durable immunity was established evident by responses lasting up to 10 years in some patients [21]. The mechanisms underpinning the in vivo efficacy of CTLA-4 blockade remains controversial. Targeting both effector $\mathrm{T}$ cells and Tregs appear critical for maximal anti-tumor activity [22]. However, antiCTLA-4 activity has also been linked to Fc mediated Treg depletion at the tumor site by tumor infiltrating macrophages [23, 24].

\section{Blockade of PD-1/PD-L1 axis expanding boundaries}

Similar to CTLA-4, Programmed cell death 1 (PD-1) is not expressed on naïve or memory $\mathrm{T}$ cells but is expressed on activated and exhausted $\mathrm{T}$ cells $[25,26]$. PD-1 has two binding partners, Programmed cell death ligand 1 (PD-L1) and Programmed cell death ligand 2 (PD-L2). Though the role of PD-L2 as a target in immuno-oncology has yet to be clarified [27, 28], ample pre-clinical and clinical data demonstrate the relevance of PD-L1. PD-L1 is expressed on dendritic cells, macrophages, myeloid-derived suppressor cells (MDSCs), fibroblasts, and $\mathrm{T}$ cells [28-30]. PD-L1 has also been detected in multiple human cancer cells including breast cancer, colon cancer, glioblastoma, non-small cell lung cancer, melanoma, and ovarian cancers, among others [29]. In addition, some tumors such as melanoma express PD-1 which can directly promote tumorigenesis in a cell-intrinsic fashion by modulating mTOR pathways. Therefore, anti-PD-1 antibodies can also directly inhibit tumor growth [31].
Unlike CTLA-4, PD-1/PD-L1 engagement does not interfere with co-stimulation but negatively regulates anti-tumor immunity through apoptosis and inhibition of $\mathrm{T}$ cell signaling. Tumor cell expression of $\mathrm{PD}-\mathrm{L} 1$ ligation triggers apoptosis of activated $\mathrm{T}$ cells leading to the loss of $\mathrm{T}$ cells in vivo and subsequent outgrowth of tumor [32-34]. Ligation of PD-L1 and PD-1 negatively regulates $\mathrm{T}$ cell activity by multiple ways. The cytoplasmic domain of PD-1 includes a conventional immunoreceptor tyrosine-based inhibition motif (ITIM) and immunoreceptor tyrosinebased switch motif (ITSM) which bind inhibitory tyrosine phosphatase SHP-2 [33], disrupts TCRinduced stop signal [35], and promotes $\mathrm{T}$ cell motility to limit the time of cognate interactions [36].

In contrast to CTLA- $4^{-1-}$ mice, PD-L1 ${ }^{-1-}$ [32] and PD$1^{-/}[37,38]$ mice do not develop massive, lethal lymphoproliferative disease. Instead, there is only organspecific inflammation further supporting the fact that CTLA-4 and PD-1 function at different stages of immune activation.

The prior success of anti-CTLA-4 led to accelerated development of antibodies targeting PD-1 [39, 40] and PD-L1 [41]. Studies testing these new drugs uncovered several distinctions from anti-CTLA-4 therapy. In addition to enhanced efficacy in melanoma [39-41], disrupting the PD-1 pathway demonstrated efficacy in non small cell lung cancer [39, 41, 42], which was previously thought to be refractory to immunotherapy. Second, treatment with drugs blocking PD-1/PD-L1 caused fewer immune related adverse events than anti-CTLA-4, which is consistent with the less severe inflammatory phenotype of PD-1 and PD-L1 deficient mice compared to that of CTLA-4 deficient mice. Several drugs targeting either PD-1 (Nivolumab, Bristol-Myers Squibb; Pembrolizumab, Merck) or PD-L1 (Atezolizumab, Roche) have received accelerated FDA approval for various applications and more are in development.

\section{Maximizing response with combination therapy}

Though a subset of patients responds to NCR blockade as monotherapy, the majority of patients do not respond to the release of a single axis. Thus, combination therapy targeting multiple pathways may provide additional support to the development of anti-tumor host immunity. Indeed, combination of drugs targeting CTLA-4 or the PD-1/PD-L1 pathway led to synergistic efficacy versus monotherapy in both murine models and patients [43-45] leading to FDA approval of combination Ipilimumab and Nivolumab for advanced melanoma.

Synergist clinical activity with combination therapy indicate non-redundant mechanisms of action for 
anti-CTLA-4 and anti-PD-1, which may be attributed to differential regulatory mechanisms dictating target expression. Expression of CTLA-4 is induced by TCR signaling [9] and surface expression is rapidly upregulated by mobilizing large intracellular pools of CTLA4. Combined with the observation that its ligands CD80 and CD86 are expressed on APCs, it is believed that the CTLA-4 axis works to restrain T cells within secondary lymphoid organs where $\mathrm{T}$ cell activation occurs $[1,19,20]$. In contrast to CTLA-4, it is viewed that PD-1/PD-L1 pathway acts to restrain $\mathrm{T}$ cell responses in the peripheral tissues, such as at the tumor bed where ligand and receptor are both in abundance $[1,34,46,47]$. PD-L1 can be upregulated by tumor cell oncogenes [48-53] or by other mediators highly expressed within the tumor microenvironment (TME) such as tumor hypoxia [30], TLR-mediated signaling pathways [54], and IFNY [55]. As a result, future studies identifying regulatory mechanisms governing NCR expression may help guide strategic combination therapy and also aid in selecting patients to optimize treatment protocol.

Another approach to improving the response to NCR blockade, is reversing mechanisms which limit its efficacy. There are multiple mediators which subvert the full potential of immunotherapy such as tumor-infiltrating MDSCs. MDSCs are a group of heterogeneous, immature myeloid cells that are aggressively expanded and pathologically activated by tumor-derived factors [56]. MDSCs exert suppression over $\mathrm{T}$ cells through multiple mechanisms including the production of reactive oxygen species, nitric oxide, and arginase [57]; ultimately leading to $\mathrm{T}$ cell suppression [58] and increased tumor burden [57].

Importantly, the accumulation of MDSCs within the tumor bed has emerged as an important mechanism of resistance to immunotherapy such as NCR blockade. In combination anti-PD-1 and anti-CTLA-4 in B16 melanoma, reduced treatment efficacy was observed when MDSCs were recruited via IDO overexpression (B16-IDO) by the tumor $[59,60]$ and blocking MDSCs recruitment via CSF-1R blockade improved efficacy in B16-IDO but not in B16. In CT26 colon carcinoma model, characterized by its aggressive MDSC compartment [61], MDSC depletion enhanced the efficacy of the combination therapy of anti-CTLA-4 and anti-PD-1. While late intervention of anti-PD-1 and anti-CTLA-4 on large, established CT26 colon cancer and 4 T1 breast cancer did not have any therapeutic efficacy, supplementing the combination therapy with 5-azacytidine and etinostat to inhibit MDSCs, sensitized tumors to NCR blockade leading to tumor reduction [62]. Together, these studies clearly demonstrate that targeting MDSCs as part of NCR blockade may provide an additional dimension to therapeutic efficacy.

\section{VISTA: a new horizon in NCR blockade}

VISTA, also known as c10orf54, PD-1H [63, 64], DD1 $\alpha$ [65], Gi24 [66], Dies1 [67], and SISP1 [68] is a member of the B7 family of NCRs and represents a new target for immunotherapy. Murine VISTA is a type I transmembrane protein with a single IgV domain with sequence homology to its B7 relatives with conserved segments thought to be critical for the IgV stability [69]. However, VISTA also has unique features such as additional cysteine residues in the Ig-V domain, an insertion of a long loop between the C" and D strands, and the absence of a second Ig domain in the ectodomain [69]. These features suggest that VISTA could function as a receptor as well as a ligand. Indeed, structural modeling suggest homology to either PD-1 [63] or PD-L1 [69]. However, VISTA does not cluster with the B7 family at standard confidence limits, suggesting that it is only weakly associated with this family [69]. VISTA is the most conserved among the B7 members. Human VISTA shares $78 \%$ identity with murine VISTA according to a Global Alignment Search Tool, an unprecedented sequence identity among NCRs. By comparison, human and murine PD-L1, PD-L2, CTLA-4 and PD-1 share $70 \%, 63 \%, 76 \%$, and $59 \%$ sequence identity, respectively. In particular, the cytoplasmic tail of VISTA is highly conserved in mice and humans with $86.5 \%$ identity and VISTA's unique features in the extracellular domain are also highly conserved.

The surface expression pattern of VISTA is clearly distinct from CTLA-4, PD-1, and PD-L1. [1] VISTA is expressed on naïve $T$ cells [69] whereas $P D-1$ and CTLA-4 are not, which may suggest that VISTA functions to restrain $\mathrm{T}$ cell activity at an even earlier stage in $\mathrm{T}$ cell priming. [2] VISTA is expressed on both T cells and APCs with very high expression on myeloid cells [69]. [3] VISTA is hematopoietically restricted and in multiple cancer models, VISTA was only detected on tumor infiltrating leukocytes and not on tumor cells [70]. This unique surface expression pattern suggests that VISTA may function to restrict $\mathrm{T}$ cell immunity at different stages compared to PD-1/ PD-L1 and CTLA-4 axes. Importantly, the pattern of VISTA expression is remarkably similar between human and mice (Table 1). Interestingly, VISTA is abundantly stored within intracellular compartments (unpublished), similar to CTLA-4. In fact, the intracellular CTLA-4 compartment is a key component in the stringent regulation of surface CTLA-4. Intracellular CTLA-4 is mobilized to the surface following T cell stimulation in a magnitude which is directly proportional to TCR signaling [5, 71-73]. Comparably, the 
majority of VISTA is detected within the intracellular compartment of myeloid cells with surface VISTA rapidly endocytosed (unpublished). However, steady, but high, levels of VISTA are maintained on the cellular surface of myeloid cells [69, 70, 74]. Studies investigating the mechanisms underlying intracellular and surface VISTA expression are ongoing.

VISTA has been demonstrated to exert both ligand and receptor functions. First, VISTA can function as a ligand to negatively regulate $\mathrm{T}$ cell activation. In vitro, VISTA on APCs and cell-free VISTA-Ig fusion protein (extracellular domain of VISTA fused with human IgG1 Fc) inhibited $\mathrm{CD}^{+}$and $\mathrm{CD} 4^{+} \mathrm{T}$ cell at the early stage of activation indicated by suppression of CD69, CD25, CD44, and CD62L [69]. VISTA Ig suppressed the production of IL-2 and IFN $\gamma$ in both $\mathrm{CD} 4^{+}$naïve and memory $\mathrm{T}$ cells as well as in $\mathrm{CD}^{+} \mathrm{T}$ cells [69]. Unlike the PD-1/PD-L1 pathway, VISTA does not directly regulate $\mathrm{T}$ cell response by the induction of apoptosis [69]. VISTA-Ig fusion protein also promotes in vitro conversion of naïve $\mathrm{CD}^{+} \mathrm{T}$ cells to Tregs in both mouse and human [74, 75]. VISTA expression on Tregs also contributes to the suppression of $\mathrm{T}$ cell proliferation in in vitro suppression assay [70]. Finally, in mice that were vaccinated with irradiated MCA105 tumor cells to generate immunity, re-challenge with VISTA-overexpressing MCA105 tumor cells lead enhanced tumor growth compared to re-challenge with the VISTA negative parent MCA105 indicating that VISTA expression can overcome protective antitumor immunity [69].

Second, VISTA has been demonstrated to function as a receptor on $\mathrm{T}$ cells which negatively regulates their activity. $\mathrm{VISTA}^{-1-} \mathrm{CD} 4^{+} \mathrm{T}$ cells respond more vigorously than wild type (WT) $\mathrm{CD}^{+} \mathrm{T}$ cells to both polyclonal and antigen specific stimulation leading to

Table 1 VISTA expression levels on human and murine subsets as evaluated by FACS analysis

\begin{tabular}{lll}
\hline Cell Type & \multicolumn{2}{l}{ Surface VISTA expression } \\
\cline { 2 - 3 } & Human & Mouse \\
\hline $\mathrm{CD}^{+}$naïve T cells & + & ++ \\
$\mathrm{CD}^{+}$FoxP3 ${ }^{+} \mathrm{T}_{\text {reg }}$ & + & ++ \\
$\mathrm{CD}^{+}$memory T cells & + & ++ \\
$\mathrm{CD}^{+}$T cells & + & + \\
B cells & - & - \\
NK cells & - & - \\
Peritoneal macrophages & N/D & +++ \\
Monocytes & +++ & +++ \\
Neutrophils & +++ & +++ \\
Dendritic cells & +++ & +++ \\
\hline
\end{tabular}

Adapted from Wang et al. and Lines et al increased proliferation and production of IFN $\gamma, \mathrm{TNF} \alpha$, and IL-17A [64]. In addition, VISTA ${ }^{-1-} \mathrm{T}$ cells induce exacerbated graft-versus-host disease (GVHD) compared to WT T cells when transferred into F1 recipients [76]. When WT CD4 ${ }^{+} \mathrm{T}$ cells are stimulated in vitro or in vivo in the absence of VISTA on APCs, an anti-VISTA agonist antibody (mam82), which can only target VISTA on the $\mathrm{T}$ cell, reduces antigen specific activation [64]. Finally, another anti-VISTA agonist antibody (MH5A) prevents the development of GVHD induced by WT T cells [63] but is ineffective when disease is induced by VISTA ${ }^{-1-} \mathrm{T}$ cells [76]. In addition to $\mathrm{T}$ cells, VISTA can also function as a receptor on myeloid cells. Transfection of monocytes from healthy donors to overexpress VISTA led to the spontaneous secretion of inflammatory cytokines IL-8, IL-1 $\beta$, IL-6, TNF $\alpha$, and IL-10 [77]. In HIV positive patients, infected monocytes expressed higher amounts of VISTA than healthy monocytes and also spontaneously expressed more TNF $\alpha$, IL-1 $\beta$, and IL6 mRNA than healthy monocytes did [77]. VISTA transfected HIV-infected monocytes induced enhanced IFN $\gamma$ production by antigen-specific autologous $\mathrm{T}$ cells compared to vector control and silenced VISTA [77]. In this transfection system with HIV infected monocytes, VISTA-mediated myeloid activation and subsequent $\mathrm{T}$ cell activation overshadowed VISTAdriven immunosuppressive functions. VISTA highly conserved cytoplasmic tail does not contain any classic signaling motif. However, it contains potential protein kinase $C$ binding sites as well as proline residues that could function as docking sites for adaptor proteins. In addition, it contains multiple potential serine, threonine, and tyrosine phosphorylation sites. Importantly, the transfection of monocyte with cytoplasmic tail-deficient VISTA abrogated the spontaneous elaboration of cytokine [77], suggesting that signaling through VISTA is both possible and required. The apparent opposing functions of VISTA $\mathrm{T}$ cells and monocytes is unresolved and requires further investigation. One possible explanation for this discrepancy is the dysregulated level of VISTA expression in transfected or HIV infected monocytes. Other negative checkpoints inhibitors have been associated with positively or negatively regulating innate cells activity depending on their expression level [78].

In multiple mouse models, VISTA expression is upregulated in the TME and plays a critical role in shaping anti-tumor immunity [70]. Distinct from PDL1, VISTA expression is restricted to the tumorinfiltrating leukocytes and was not detected on tumor cells. In particular, VISTA expression is specifically upregulated on tumor infiltrating myeloid cells such as myeloid DCs and MDSCs, and on tumor 
infiltrating Tregs compared to those in the periphery [70]. On MDSCs, VISTA increased almost 10-fold on tumor-infiltrating leukocytes compared to those found in the peripheral lymph node [70]. Importantly, this indicates that tumors with infiltrating immune cells and especially MDSCs may harbor abundant levels of VISTA available for therapeutic targeting.

Anti-VISTA monotherapy reduced tumor growth in multiple pre-clinical models, B16OVA melanoma, B16BL6 melanoma, MB49 bladder carcinoma, and PTEN/ BRAF inducible melanoma [70]. In all models, antiVISTA enhanced $T$ cell response within the TME as well as systemically leading to increased accumulation, proliferation, CD44 expression, and IFN $\gamma$ and TNF $\alpha$ production [70]. Additionally, VISTA blockade reduced natural Treg mediated suppression of $\mathrm{T}$ cells and diminished tumor-induced differentiation of Tregs [70]. Finally, anti-VISTA reduced tumor-infiltrating MDSCs in the B16OVA and PTEN/BRAF melanoma models [70]. Since abnormal myelopoiesis and accumulation of MDSCs are characteristic of many tumors [79], normalizing the myeloid component offers a new opportunity for immunomodulation by anti-VISTA, a mechanism distinct from other forms of NCR blockade. An anti-human VISTA antibody is currently in phase I clinical trial for evaluation in patients with non-small cell lung cancer among other cancers. The mouse surrogate of antihuman VISTA antibody demonstrated tumor growth inhibition by modulating of the myelomonocytic and T cell compartments in human VISTA knock-in mice [80].
Important to its clinical potential as a therapeutic target, some aspect of VISTA mediated regulation are unique and therefore, targeting VISTA synergizes with the mechanism of actions of the NCRs currently targeted in the clinic (Fig. 1). Combination antibody blockade (anti-VISTA and anti-PD-1 or anti-PD-L1) synergistically enhanced $\mathrm{T}$ cell responses [81]. Ultimately, combination therapy led to reduced tumor growth, enhanced survival, increased IFN $\gamma$, tumor necrosis factor (TNF $\alpha$ ), and Granzyme B within $\mathrm{CD}^{+} \mathrm{T}$ cells further supporting a mechanism of action non-redundant to established NCR blockade in clinic.

\section{Conclusion}

Identification of NCRs as critical mechanisms limiting T cell response and the use of monoclonal antibody blockade to support the development of persistent $\mathrm{T}$ cell immunity in the setting of cancer have transformed cancer therapy. Lessons learned from anti-CTLA-4 and antiPD-1 pathway blockade have encouraged continued discovery and development of NCR blockade while revealing additional opportunities for improvement. As combination immunotherapy continues to rise to the forefront of cancer treatment, targeting VISTA may offer a particularly attractive and unique opportunity for synergism due to its role in restricting very early $\mathrm{T}$ cell activation events and high expression on tumor infiltrating MDSCs suggesting that anti-VISTA pathway blockade may occupy a distinct therapeutic compartment.

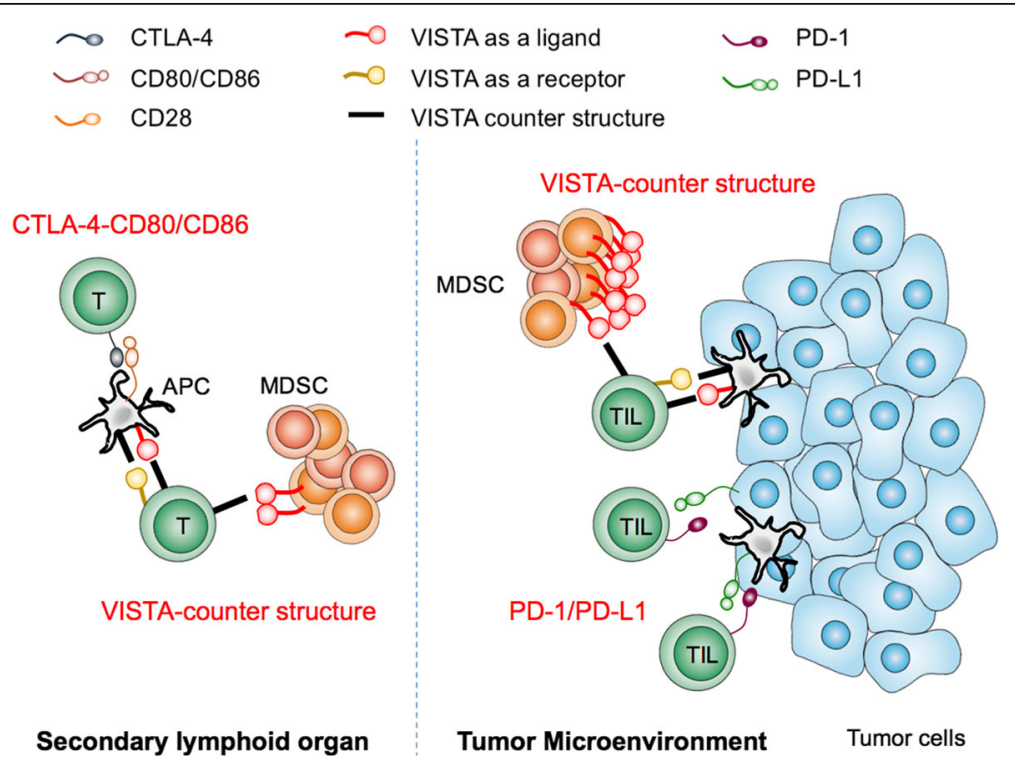

Fig. 1 VISTA functions non-redundantly to NCRs currently targeted in clinic. Each NCR occupies distinct temporal and spatial opportunities for blockade to release T cell suppression: [1] VISTA, as a receptor on T cells inhibit early T cell activation while [2] CTLA-4/CD80-86 interaction inhibit post-TCR signaling in secondary lymphoid organs. [3] PD-1/PD-L1 interaction inhibits effector T cells in inflamed tumor tissue. [4] VISTA, as a ligand on MDSCs engages counter structure to inhibit T cells in tumor tissue and secondary lymphoid organs 


\section{Abbreviations}

APCs: Antigen presenting cells; CSF-1R: Colony stimulating factor 1 receptor; CTLA-4: Cytotoxic T Lymphocyte Antigen 4; FDA: Food and Drug Administration; GVHD: Graft-versus-host disease; HIV: Human immunodeficiency virus; IDO: Indoleamine 2,3-dioxygenase; ITIM: Immunoreceptor tyrosine-based inhibition motif; ITSM: Immunoreceptor tyrosine-based switch motif; MDSCs: Myeloid-derived suppressor cells; NCR: Negative checkpoint regulator; nTregs: Natural regulatory T cells; PD 1: Programmed cell death 1; PD-L1: Programmed cell death ligand 1; PDL2: Programmed cell death ligand 2; TCR: T cell receptor; TLR: Toll Like receptor; TME: Tumor microenvironment; VISTA: V-domain Ig Suppressor of T Cell Activation; WT: Wild type

\section{Acknowledgements}

Not applicable.

\section{Funding}

National Institutes of Health grant 1R01Al098007 (RJN)

Research contract from ImmuNext (RJN)

DF fund philanthropic support (JD)

\section{Availability of supporting data}

Not applicable.

\section{Authors' contributions}

JD, ILM and AK wrote the manuscript with JD being a major contributor. RJN reviewed and edited the manuscript. All authors read and approved the final manuscript.

\section{Competing interests}

R.J. Noelle is CSO, has commercial research grant, other commercial research support, ownership interest (including patents), and is a consultant/advisory board member of ImmuNext. I. Le Mercier is a Scientist and has ownership interest (including patents) in ImmuNext. The other authors declare that they have no competing interests.

\section{Consent for publication}

Not applicable.

\section{Ethics approval and consent to participate} Not applicable.

\section{Author details}

'Department of Microbiology and Immunology, Geisel School of Medicine at Dartmouth, Norris Cotton Cancer Center, One Medical Center Drive, Room 730, Lebanon, NH 03756, USA. ${ }^{2}$ ImmuNext, Inc., One Medical Center Drive, Lebanon, NH 03756, USA.

Received: 12 July 2016 Accepted: 7 November 2016

Published online: 20 December 2016

\section{References}

1. Topalian SL, Drake CG, Pardoll DM. Immune checkpoint blockade: a common denominator approach to cancer therapy. Cancer Cell. 2015;27(4): 450-61.

2. Pentcheva-Hoang T, Egen JG, Wojnoonski K, Allison JP. B7-1 and B7-2 selectively recruit CTLA-4 and CD28 to the immunological synapse. Immunity. 2004;21(3):401-13.

3. Lenschow DJ, Walunas TL, Bluestone JA. CD28/B7 system of T cell costimulation. Annu Rev Immunol. 1996;14:233-58.

4. van der Merwe PA, Bodian DL, Daenke S, Linsley P, Davis SJ. CD80 (B7-1) binds both CD28 and CTLA-4 with a low affinity and very fast kinetics. J Exp Med. 1997;185(3):393-403.

5. Alegre ML, et al. Regulation of surface and intracellular expression of CTLA4 on mouse T cells. J Immunol. 1996;157(11):4762-70.

6. Rudd CE, Martin M, Schneider H. CTLA-4 negative signaling via lipid rafts: a new perspective. Sci STKE. 2002;2002(128):e18

7. Lee KM, et al. Molecular basis of T cell inactivation by CTLA-4. Science. 1998; 282(5397):2263-6.

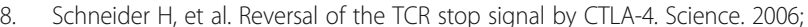
313(5795):1972-5
9. Brunner MC, et al. CTLA-4-Mediated inhibition of early events of $\mathrm{T}$ cell proliferation. J Immunol. 1999:162(10):5813-20.

10. Grohmann U, et al. CTLA-4-Ig regulates tryptophan catabolism in vivo. Nat Immunol. 2002;3(11):1097-101.

11. Fallarino $F$, et al. Modulation of tryptophan catabolism by regulatory $T$ cells. Nat Immunol. 2003:4(12):1206-12.

12. Qureshi OS, et al. Trans-endocytosis of CD80 and CD86: a molecular basis for the cell-extrinsic function of CTLA-4. Science. 2011:332(6029):600-3.

13. Tai $X$, et al. Basis of CTLA-4 function in regulatory and conventional CD4(+) T cells. Blood. 2012;119(22):5155-63.

14. Tivol EA, et al. Loss of CTLA-4 leads to massive lymphoproliferation and fatal multiorgan tissue destruction, revealing a critical negative regulatory role of CTLA-4. Immunity. 1995:3(5):541-7.

15. Waterhouse $P$, et al. Lymphoproliferative disorders with early lethality in mice deficient in Ctla-4. Science 1995:270(5238):985-8.

16. Wing $\mathrm{K}$, et al. CTLA-4 control over Foxp3+ regulatory $T$ cell function. Science. 2008:322(5899):271-5.

17. Leach DR, Krummel MF, Allison JP. Enhancement of antitumor immunity by CTLA-4 blockade. Science. 1996:271(5256):1734-6.

18. Hurwitz AA, Yu TF, Leach DR, Allison JP. CTLA-4 blockade synergizes with tumor-derived granulocyte-macrophage colony-stimulating factor for treatment of an experimental mammary carcinoma. Proc Natl Acad Sci U S A. 1998:95(17):10067-71.

19. Hodi FS, et al. Improved survival with ipilimumab in patients with metastatic melanoma. N Engl J Med. 2010;363(8):711-23.

20. Robert $\mathrm{C}$, et al. Ipilimumab plus dacarbazine for previously untreated metastatic melanoma. N Engl J Med. 2011;364(26):2517-26.

21. Schadendorf $D$, et al. Pooled analysis of long-term survival data from phase II and phase III trials of ipilimumab in unresectable or metastatic melanoma. J Clin Oncol. 2015:33(17):1889-94.

22. Peggs KS, Quezada SA, Chambers CA, Korman AJ, Allison JP. Blockade of CTLA-4 on both effector and regulatory $T$ cell compartments contributes to the antitumor activity of anti-CTLA-4 antibodies. J Exp Med. 2009:206(8):1717-25.

23. Simpson TR, et al. Fc-dependent depletion of tumor-infiltrating regulatory $T$ cells co-defines the efficacy of anti-CTLA-4 therapy against melanoma. J Exp Med. 2013;210(9):1695-710

24. Selby MJ, et al. Anti-CTLA-4 antibodies of lgG2a isotype enhance antitumor activity through reduction of intratumoral regulatory $T$ cells. Cancer Immunol Res. 2013;1(1):32-42.

25. Agata $Y$, Kawasaki A, Nishimura $H$, Ishida $Y$, Tsubata $T$, Yagita $H$, et al. Expression of the PD-1 antigen on the surface of stimulated mouse $T$ and $B$ lymphocytes. Int. Immunol. 1996;8:765-72.

26. Day $\mathrm{CL}$, et al. PD-1 expression on HIV-specific T cells is associated with T-cell exhaustion and disease progression. Nature. 2006:443(7109):350-4

27. Taube JM, et al. Association of PD-1, PD-1 ligands, and other features of the tumor immune microenvironment with response to anti-PD-1 therapy. Clin Cancer Res. 2014;20(19):5064-74

28. Zou W, Wolchok JD, Chen L. PD-L1 (B7-H1) and PD-1 pathway blockade for cancer therapy: Mechanisms, response biomarkers, and combinations. Sci Transl Med. 2016:8(328):328rv4

29. Zou W, Chen L. Inhibitory B7-family molecules in the tumour microenvironment. Nat Rev Immunol. 2008:8(6):467-77.

30. Noman MZ, et al. PD-L1 is a novel direct target of HIF-1a, and its blockade under hypoxia enhanced MDSC-mediated T cell activation. J Exp Med. 2014 211(5):781-90.

31. Kleffel S, et al. Melanoma cell-intrinsic PD-1 receptor functions promote tumor growth. Cell. 2015;162(6):1242-56.

32. Dong $\mathrm{H}$, et al. B7-H1 determines accumulation and deletion of intrahepatic CD8(+) T lymphocytes. Immunity. 2004;20(3):327-36

33. Chemnitz JM, Parry RV, Nichols KE, June CH, Riley JL. SHP-1 and SHP-2 associate with immunoreceptor tyrosine-based switch motif of programmed death 1 upon primary human T cell stimulation, but only receptor ligation prevents T cell activation. J Immunol. 2004;173(2):945-54.

34. Dong $\mathrm{H}$, et al. Tumor-associated B7-H1 promotes T-cell apoptosis: a potential mechanism of immune evasion. Nat Med. 2002;8(8):793-800.

35. Fife BT, et al. Interactions between PD-1 and PD-L1 promote tolerance by blocking the TCR-induced stop signal. Nat Immunol. 2009;10(11):1185-92.

36. Honda $T$, et al. Tuning of antigen sensitivity by $T$ cell receptor-dependent negative feedback controls T cell effector function in inflamed tissues. mmunity. 2014;40(2):235-47. 
37. Nishimura T, Ohta A. A critical role for antigen-specific Th1 cells in acute liver injury in mice. J Immunol. 1999;162(11):6503-9.

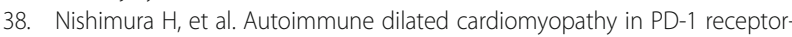
deficient mice. Science. 2001;291(5502):319-22.

39. Topalian SL, et al. Safety, activity, and immune correlates of anti-PD-1 antibody in cancer. N Engl J Med. 2012;366(26):2443-54.

40. Hamid O, et al. Safety and tumor responses with lambrolizumab (anti-PD-1) in melanoma. N Engl J Med. 2013;369(2):134-44.

41. Brahmer JR, et al. Safety and activity of anti-PD-L1 antibody in patients with advanced cancer. N Engl J Med. 2012;366(26):2455-65.

42. Topalian SL, et al. Survival, durable tumor remission, and long-term safety in patients with advanced melanoma receiving nivolumab. J Clin Oncol. 2014; 32(10):1020-30.

43. Curran MA, Montalvo W, Yagita H, Allison JP. PD-1 and CTLA-4 combination blockade expands infiltrating $T$ cells and reduces regulatory $T$ and myeloid cells within B16 melanoma tumors. Proc Natl Acad Sci U S A. 2010;107(9): 4275-80.

44. Postow MA, et al. Nivolumab and ipilimumab versus ipilimumab in untreated melanoma. N Engl J Med. 2015;372(21):2006-17.

45. Wolchok JD, et al. Nivolumab plus ipilimumab in advanced melanoma. $N$ Engl J Med. 2013;369(2):122-33.

46. Chen L, Han X. Anti-PD-1/PD-L1 therapy of human cancer: past, present, and future. J Clin Invest. 2015;125(9):3384-91.

47. Ahmadzadeh $M$, et al. Tumor antigen-specific CD8 T cells infiltrating the tumor express high levels of PD-1 and are functionally impaired. Blood. 2009;114(8):1537-44

48. Pardoll DM. The blockade of immune checkpoints in cancer immunotherapy. Nat Rev Cancer. 2012;12(4):252-64.

49. Crane CA, et al. PI(3) kinase is associated with a mechanism of immunoresistance in breast and prostate cancer. Oncogene. 2009;28(2):306-12.

50. Parsa AT, et al. Loss of tumor suppressor PTEN function increases B7-H1 expression and immunoresistance in glioma. Nat Med. 2007;13(1):84-8.

51. Marzec M, et al. Oncogenic kinase NPM/ALK induces through STAT3 expression of immunosuppressive protein CD274 (PD-L1, B7-H1). Proc Natl Acad Sci U S A. 2008;105(52):20852-7.

52. Akbay EA, et al. Activation of the PD-1 pathway contributes to immune escape in EGFR-driven lung tumors. Cancer Discov. 2013;3(12):1355-63.

53. Steidl C, et al. MHC class II transactivator CIITA is a recurrent gene fusion partner in lymphoid cancers. Nature. 2011;471(7338):377-81.

54. Ritprajak P, Azuma M. Intrinsic and extrinsic control of expression of the immunoregulatory molecule PD-L1 in epithelial cells and squamous cell carcinoma. Oral Oncol. 2015;51(3):221-8.

55. Taube JM, et al. Colocalization of inflammatory response with B7-h1 expression in human melanocytic lesions supports an adaptive resistance mechanism of immune escape. Sci Transl Med. 2012;4(127):127ra37.

56. Condamine T, Mastio J, Gabrilovich DI. Transcriptional regulation of myeloid-derived suppressor cells. J Leukoc Biol. 2015;98(6):913-22.

57. Talmadge JE, Gabrilovich DI. History of myeloid-derived suppressor cells. Nat Rev Cancer. 2013;13(10):739-52.

58. Gabrilovich DI, Nagaraj S. Myeloid-derived suppressor cells as regulators of the immune system. Nat Rev Immunol. 2009;9(3):162-74.

59. Holmgaard RB, et al. Tumor-expressed IDO recruits and activates MDSCs in a treg-dependent manner. Cell Rep. 2015;13(2):412-24.

60. Holmgaard RB, Zamarin D, Lesokhin A, Merghoub T. Targeting myeloidderived suppressor cells with colony stimulating factor-1 receptor blockade can reverse immune resistance to immunotherapy in .... EBioMedicine. 2016;6:50-8. doi:10.1016/j.ebiom.2016.02.024

61. Youn J-I, Nagaraj S, Collazo M, Gabrilovich DI. Subsets of myeloid-derived suppressor cells in tumor-bearing mice. J Immunol. 2008;181(8):5791-802.

62. Kim K, et al. Eradication of metastatic mouse cancers resistant to immune checkpoint blockade by suppression of myeloid-derived cells. Proc Natl Acad Sci U S A. 2014;111(32):11774-9.

63. Flies DB, Wang $\mathrm{S}, \mathrm{Xu} \mathrm{H}$, Chen L. Cutting edge: A monoclonal antibody specific for the programmed death-1 homolog prevents graft-versus-host disease in mouse models. J Immunol. 2011;187(4):1537-41.

64. Flies DB, et al. Coinhibitory receptor PD-1H preferentially suppresses CD4 ${ }^{+} \mathrm{T}$ cell-mediated immunity. J Clin Invest. 2014;124(5):1966-75.

65. Yoon KW, et al. Control of signaling-mediated clearance of apoptotic cells by the tumor suppressor p53. Science. 2015;349(6247):1261669.
66. Sakr MA, et al. Gl24 enhances tumor invasiveness by regulating cell surface membrane-type 1 matrix metalloproteinase. Cancer Sci. 2010; 101(11):2368-74.

67. Aloia L, Parisi S, Fusco L, Pastore L, Russo T. Differentiation of embryonic stem cells 1 (Dies 1 ) is a component of bone morphogenetic protein 4 (BMP4) signaling pathway required for proper differentiation of mouse embryonic stem cells. J Biol Chem. 2010;285(10):7776-83.

68. SISP-1, a novel p53 target gene and use thereof. https://www.google.com/ patents/US7655778.

69. Wang $L$, et al. VISTA, a novel mouse Ig superfamily ligand that negatively regulates T cell responses. J Exp Med. 2011;208(3):577-92.

70. Le Mercier I, et al. VISTA regulates the development of protective antitumor immunity. Cancer Res. 2014;74(7):1933-44.

71. Linsley PS, et al. Intracellular trafficking of CTLA-4 and focal localization towards sites of TCR engagement. Immunity. 1996:4(6):535-43.

72. Gibson HM, et al. Induction of the CTLA-4 gene in human lymphocytes is dependent on NFAT binding the proximal promoter. J Immunol. 2007; 179(6):3831-40

73. Li J, et al. Regulation of cytotoxic T lymphocyte antigen 4 by cyclic AMP. Am J Respir Cell Mol Biol. 2013;48(1):63-70.

74. Lines $J$, et al. VISTA is an immune checkpoint molecule for human T cells. Cancer Res. 2014;74(7):1924-32.

75. Le Mercier I, Lines JL, Noelle RJ. Beyond CTLA-4 and PD-1, the generation Z of negative checkpoint regulators. Front Immunol. 2015;6:418.

76. Flies DB, Higuchi T, Chen L. Mechanistic Assessment of PD-1H Coinhibitory Receptor-Induced T Cell Tolerance to Allogeneic Antigens. J. Immunol. [published online ahead of print: April 27, 2015]; doi:10.4049/jimmunol. 1402648

77. Bharaj P, et al. Characterization of programmed death-1 homologue-1 (PD$1 \mathrm{H})$ expression and function in normal and HIV infected individuals. PLOS One. 2014;9(10):e109103.

78. Han G, Chen G, Shen B, Li Y. Tim-3: an activation marker and activation limiter of innate immune cells. Front Immunol. 2013:4:449.

79. Gabrilovich DI, Ostrand-Rosenberg S, Bronte V. Coordinated regulation of myeloid cells by tumours. Nat Rev Immunol. 2012;12(4):253-68.

80. Jiang $X$, Leu J, Singh I, Snyder LA, Wang W. Development of a mechanismbased pharmacokinetic/pharmacodynamic model to characterize tumor killing effect of an anti-VISTA monoclonal antibody in tumor .... Cancer Res. 2016; 76(14 Suppl):Abstract nr 2085. doi:10.1158/1538-7445.

81. Liu J, Yuan Y, Chen W, Putra J. Immune-checkpoint proteins VISTA and PD-1 nonredundantly regulate murine T-cell responses. PNAS. 2015;112:6682-87.

\section{Submit your next manuscript to BioMed Central and we will help you at every step:}

- We accept pre-submission inquiries

- Our selector tool helps you to find the most relevant journal

- We provide round the clock customer support

- Convenient online submission

- Thorough peer review

- Inclusion in PubMed and all major indexing services

- Maximum visibility for your research

Submit your manuscript at www.biomedcentral.com/submit 